\title{
Leiomyosarcoma of the vagina: a case report and review from the literature
}

\author{
Min Jung Suh, Dong Choon Park \\ Department of Obstetrics and Gynecology, St. Vincent's Hospital, The Catholic University of Korea, Suwon, Korea
}

\begin{abstract}
Leiomyosarcomas comprise fewer than $2 \%$ of all malignant vaginal neoplasms. Due to their rarity, treatment for vaginal leiomyosarcomas have not been determined. We describe a 66 year old woman with vaginal leiomyosarcoma, which presented as a large palpable mass with vaginal spotting. Complete surgical excision was accomplished and after surgery, she underwent radiation therapy. Tumor recurrence was not detected for the last 5 years and now her general condition is very good. This is a rare case of leiomyosarcoma arising in vagina and we report the results of successful treatment.
\end{abstract}

Key Words: Leiomyosarcoma, Vagina

\section{INTRODUCTION}

Malignant tumors in the vagina are extremely rare, comprising less than $1 \%$ of cancers of the reproductive organs. The most frequently observed vaginal tumors are squamous cell carcinomas (75-90\%), followed by adenocarcinoma (5-10\%), melanoma, and leiomyosarcoma. ${ }^{1}$ Since only about 140 leiomyosarcomas in the vagina have been reported during the past 40 years, ${ }^{2}$ guidelines for the determination of disease stage and treatment have not yet been established. Vaginal leiomyosarcomas usually originate from the smooth muscle of the vaginal wall, but they may also develop from smooth muscle cells in tissues near the vagina. Malignancy of leiomyosarcoma is diagnosed histopathologically by assessment of cellular mitosis activity and the presence of cellular dysplasia. $^{3}$

\section{CASE REPORT}

A 66-year old gravid 2-0-1-2 woman presented with vaginal spotting that had begun approximately 2 weeks earlier. Her menopause had occurred when she was at age 43 and her menstruation pattern had consisted of 30 day cycles, with normal

Received June 13, 2008, Revised July 24, 2008,

Accepted August 20, 2008

\footnotetext{
Address reprint requests to Dong Choon Park

Department of Obstetrics and Gynecology, St. Vincent's Hospital, The Catholic University of Korea, 93, Gi-dong, Paldal-gu, Suwon 442-723, Korea

Tel: 82-31-244-9504, Fax: 82-31-254-7481

E-mail: dcpark@catholic.ac.kr
}

volume and mild dysmenorrhea. She had 2 vaginal spontaneous deliveries and one abortion. Her family history was uneventful. She was recently diagnosed with asthma and was treated with oral agents for this disease.

On pelvic examination, a mass suspected of being a hematocolpos was detected, and she was transferred to the Department of Obstetrics and Gynecology of our hospital. From the results of magnetic resonance imaging (MRI), the mass was diagnosed as a uterine smooth muscle tumor suspected of being malignant or a secondary degeneration, and surgery was scheduled.

\section{Laboratory findings}

At the time of admission, her vital sign was stable and her general condition was good. Her hemoglobin concentration was $12.4 \mathrm{~g} / \mathrm{dl}$, her red blood cell volume was $37.5 \%$, her leukocyte count was $5,150 / \mathrm{mm}^{3}$, and her platelet count was $287,000 / \mathrm{mm}^{3}$, all within normal ranges. No glucose or protein was detected in her urine. Her serum concentration of CEA, SCC, and CA125 were $2.07 \mathrm{ng} / \mathrm{ml}, 0.8 \mathrm{ng} / \mathrm{ml}$, and 4.64 $\mathrm{U} / \mathrm{ml}$, respectively, all within normal ranges. Uterine cervical cytology at the time of admission was normal. Sigmoidoscopy showed that her sigmoid colon was compressed by the tumor, but it was not a rectal tumor.

\section{Radiological findings}

Pelvic examination showed that her uterus and both adnexae had become atrophic after menopause and were therefore small when palpated. An approximately $12.0 \times 6.0 \mathrm{~cm}$ sized mass was detected in the posterior vaginal wall and it was movable without tenderness. Transvaginal ultrasound also 

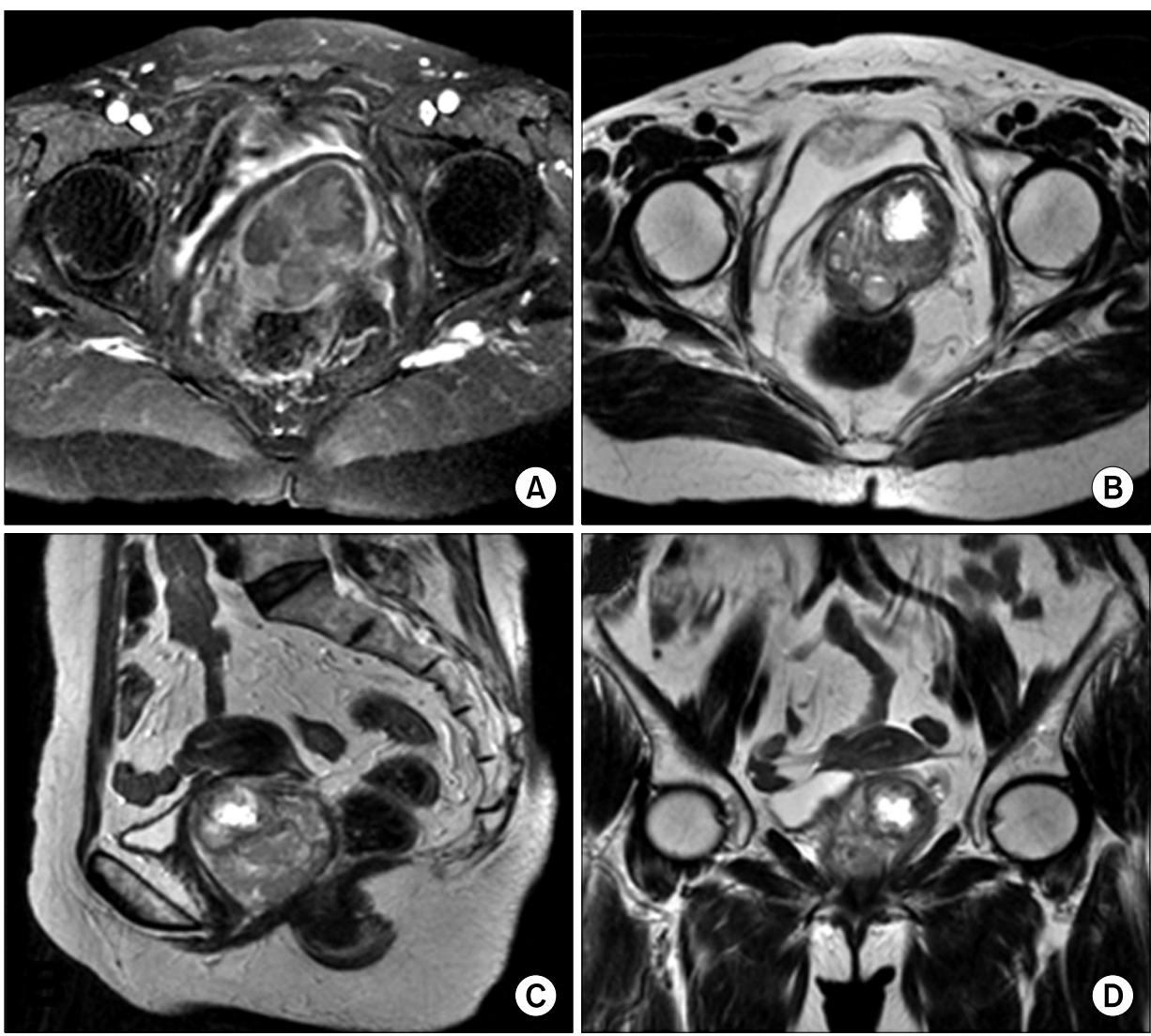

D
Fig. 1. Pre-operative MR images. They show that the about $6 \times 5.7 \times$ $5.2 \mathrm{~cm}$ sized heterogenous signal mass in posterolateral portion of the vagina. (A) T1-weighted axial MR image, (B) T2-weighted axial MR image, (C) T2-weighted sagittal MR image, (D) T2-weighted coronal MR image.
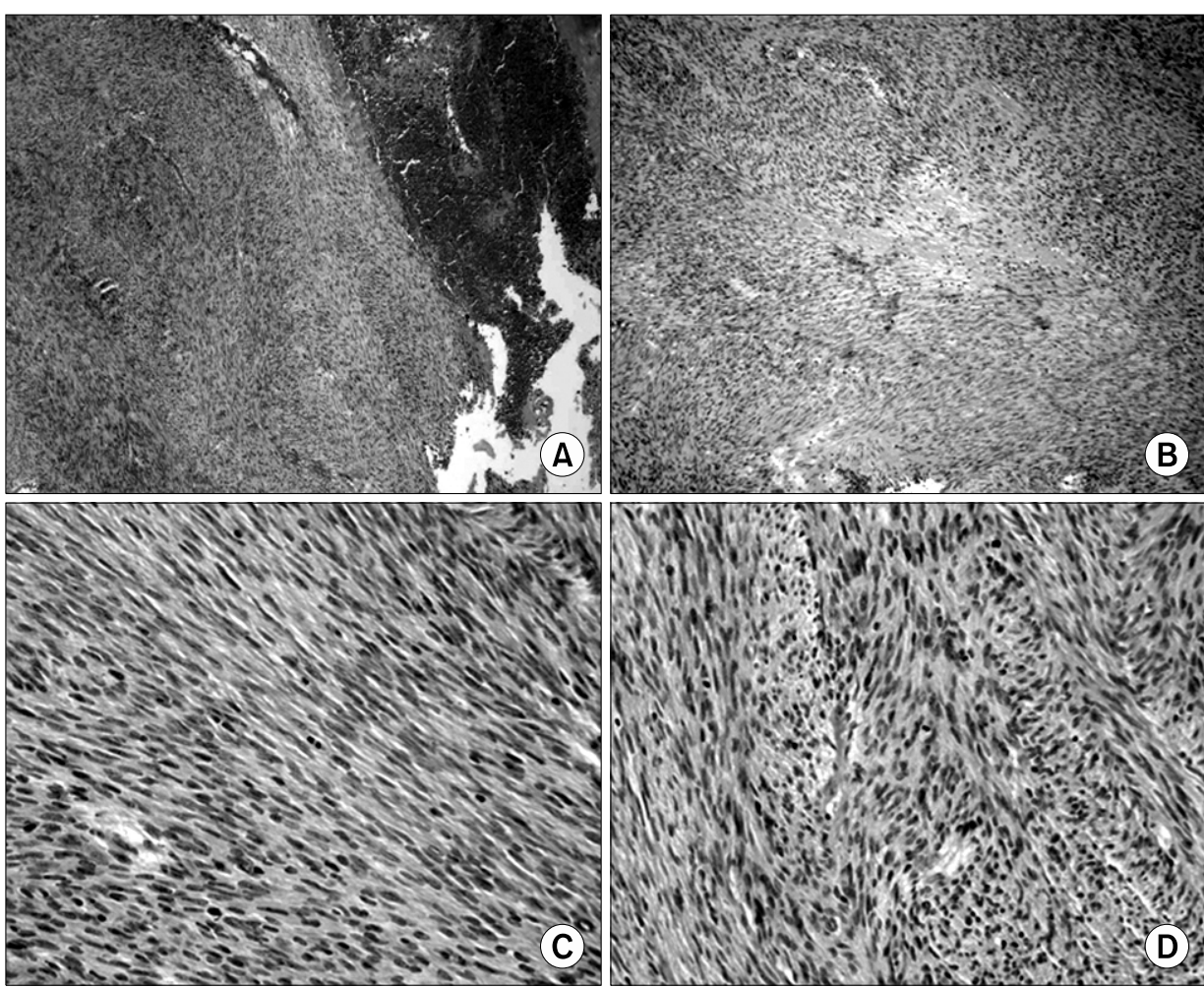

Fig. 2. (A) There are marked increased spindle cells ( $\mathrm{H} \& \mathrm{E}, \times 40)$, (B) In focal area, we could see some foci of degeneration $(\mathrm{H} \& \mathrm{E}, \times 100)$. (C, D) Spindle cells revealed moderate nuclear atypism with frequent mitosis $(\mathrm{H} \& \mathrm{E}, \times 400)$. 
showed that her uterus and both adnexae were atrophic, and that the mass in the posterior vaginal wall was approximately $6.5 \times 6.0 \mathrm{~cm}$ in size, with irregular contrast. Pelvic MRI showed a mass approximately $6 \times 5.7 \times 5.2 \mathrm{~cm}$ in volume with heterogeneous signal intensity adjacent to the rectum. The mass appeared to be leiomyoma accompanying secondary degeneration, and it could be differentiated from a smooth muscle tumor (Fig. 1).

\section{Surgical findings}

The patient was placed in the dorsal lithotomy position; spinal anesthesia was performed; the posterior vaginal wall tumor, including the capsule, was completely removed; and a drain was inserted into the area from which the tumor had been removed.

\section{Pathological findings}

Grossly, the tumor was black-brown in color and approximately $6.0 \times 5.5 \times 5.0 \mathrm{~cm}$ in size. Histologically, it was composed of spindle shaped cells, with partial secondary degeneration and necrosis. When examined at high magnification $(400 \times)$, the spindle cells showed evidence of dysplasia and frequent cell division (8.85/10 HPF). Based on histopathological results, the tumor was diagnosed as a leiomyosarcoma (Fig. 2).

\section{Surgery outcome and follow-up}

After surgery, the patient did not experience any disease related symptoms and was discharged. She underwent radiation therapy, consisting of 45 Gy external and 25 Gy intra-cavitary radiation. Tumor recurrence was not detected for the last 5-year follow-up and her general condition is good.

\section{DISCUSSION}

The criteria between benign leiomyoma and leiomyosarcoma include the level of cell division and histologic evidence of cellular dysplasia. Cell division of more than 5 of $10 \mathrm{HPF}$ is considered evidence of malignancy, in which the recurrence rate and metastatic potential is high. ${ }^{3}$

Due to their rarity, vaginal leiomyosarcomas are seldom detected prior to surgery, and typical symptoms and characteristics on physical examination have not been described. These tumors are usually diagnosed histopathologically after surgical removal. Most patients with vaginal masses have no symptoms, although some may experience vaginal or rectal bleeding, serous discharge, or, rarely, dypareunia. On physical examination, most patients show palpable nodules surrounded by normal vaginal mucosae; in patients with advanced tumors, readily breakable exophytic polypoids or necrotic vaginal masses may be palpated. These tumors may invade the rectum or pelvic tissues adjacent to the vagina, requiring histological diagnosis. ${ }^{4}$

Vaginal smooth muscle tumors most frequently develop in the anterior vaginal wall, in contrast, malignant tumors most frequently develop in the posterior vaginal wall, although they can also develop elsewhere. ${ }^{2}$ Differential diagnosis of these vaginal masses include Gartner's cyst, granuloma, epithelial inclusion cyst, other neurofibroma, rhabdomyoma, capillary hemangioma, squamous epithelial carcinoma, adenocarcinoma, rhabdomyosarcoma, melanoma, small cell carcinoma, and primary malignant tumors such as mixed müllerian sarcoma that can develop in the vagina. In addition, leiomyosarcoma has to be differentiated from gestational trophoblastic tumor, uterine endometrial cancer, and vaginal metastasis of malignant tumors developing in adjacent organs, including the uterine cervix, rectum, and bladder. ${ }^{2}$

Due to their rarity, risk for development of smooth muscle tumors have not been determined. These tumors can recur locally, and have a tendency to show hematogeneous metastasis into adjacent organs. Thus, patients with this disease have a poor prognosis, and metastasis to the lungs is frequent. ${ }^{5}$ Vaginal leiomyomas can be treated by surgical resection. Due to the possibility of future transformation to sarcoma and recurrence, as well as diagnosis as malignant after surgery, these tumors have to be removed completely. The basic treatment for vaginal smooth muscle tumor is resection of a wide area of the primary site and of the site of possible uterine sarcoma, but the extent of resection may not be related to clinical course. ${ }^{4}$ In patients treated with postoperative chemotherapy or radiation therapy, the 5 -year survival rate is only $36 \% .{ }^{4}$ The differentiation grade of the tumor is the most important prognostic factor. In addition, younger age and lower stage at the time of diagnosis and the absence of infiltration in the resection margin at the time of surgery were associated with good prognosis. ${ }^{5}$

Based on the histological results of a frozen biopsy taken during operation, our patient was diagnosed with a benign tumor suspected of secondary degeneration. However, cellular dysplasia and necrosis and 8.5/10 HFP cell division were detected in permanent section evaluation postoperatively. The mass in our patient was completely resected, and there was no infiltration into the resection margin. Nevertheless, the rarity of this disease, and findings consistent with malignancy, including cell division, cellular dysplasia, and tissue necrosis, indicated the appropriateness of treatment similar to that for vaginal smooth muscle cell tumors. Thus, following surgery, this patient underwent radiation therapy.

In summary, we describe here a patient with a leiomyosarcoma that developed in the vagina, which was treated with radiation therapy after resection. Follow-up for 5 years shows no evidence of tumor recurrence or metastasis.

\section{REFERENCES}

1. Kim BH. The vagina and vulva. In: Kim SH, McClennan BL, Outwaer EK. Radiology illustrated: Gynecologic imaging. Philadelphia: Elsevier Saunders; 2005. p.807-9. 
2. Ahram J, Lemus R, Schiavello HJ. Leiomyosarcoma of the vagina: case report and literature review. Int J Gynecol Cancer 2006; 16: 884-91.

3. Tavassoli FA, Norris HJ. Smooth muscle tumors of the vagina. Obstet Gynecol 1979; 53: 689-93.

4. Ngan HY, Fisher C, Blake P, Shepherd JH. Vaginal sarcoma: The
Royal Marsden experience. Int J Gynecol Cancer 1994; 4: 337-41.

5. Creasman WT, Phillips JL, Menck HR. The National Cancer Data Base report on cancer of the vagina. Cancer 1998; 83: 1033-40. 